\title{
Delayed gastric empting following transhiatal esophagectomy without pyloroplasty: our experience with endoscopic Botox injection
}

\begin{abstract}
Introduction: Esophagectomy is accompanied with high morbidity, in up to $70 \%$ of cases as well as significant mortality. One of the complications is delayed gastric tube emptying, up to $30 \%$. Gastric drainage procedure like pyloroplasty may solve this problem. This procedure, although relatively safe, can lead to significant complications like suture line leaks, bile reflux, and late pyloric stricture. The aim of this presentation is to demonstrate our experience in coping with this problem.
\end{abstract}

Methods: From April 2003 to June 2011, 79 patients underwent Transhiatal Esophagectomy without any drainage procedure. A retrospective analysis of the data was performed.

Results: For all patients the pylorus was intact during the operation. The most patients $\mathrm{n}=71$ had carcinoma $(\mathrm{n}=59$ adenocarcinoma, $\mathrm{n}=12$ squamous cell carcinoma). 4 patients had Achalazia, 3-caustic injury, 1-iatrogenic injury of esophagus. 33 oncological patients were treated by neo-adjuvant therapy because of locally advanced disease (T3N0-1). Stapler's cervical anastomosis was performed for 61 patients and manual for 18 patients. 12 patient developed cervical anastomotic leakage ( 9 patients after instrumental anastomosis; 3 patients after manual). 5 patients developed postoperative gastric outlet obstruction due to pylorospasm. All of them were treated successfully by endoscopic Botox injections to the "nervous" pylorus. There were no any signs of dysphagia after endotherapy in these patients in long term follow up.

Keywords: esophagectomy, pylorospasm, delayed gastric empting, Botox
Volume 4 Issue 6 - 2016

Vyacheslav Bard,' Alexander Geller, ${ }^{2}$ Michael Stein,' Hanoch Kashtan'

'Department of Surgery, Rabin Medical Center, Beilinson Hospital, Israel

${ }^{2}$ Advanced Endoscopy and EndosonographyUnit, Rabin Medical Center, Beilinson Hospital, Israel

Correspondence: Vyacheslav Bard, Rabin Medical Center, Beilinson Hospital, Petach Tikva, Israel, Email slavab@clalit.org.il

Received: July 27, 2016 | Published: September 28, 2016

\section{Introduction}

Esophagectomy is accompanied with high morbidity, in up to $70 \%$ of cases, ${ }^{1}$ as well as significant mortality. The potential postoperative complications (reflux, regurgitation, early satiety, dumping syndrome, and dysphagia) should be discussed with the patient prior to the day of surgery. Possible complications in the early postoperative period include the risk of violation of the anastomosis and pulmonary complications are caused by aspiration, which can lead to serious complications and respiratory infections. ${ }^{2}$ Many of these are caused by delayed gastric tube emptying and dilatation. The incidence of delayed gastric emptying after esophagectomy has been reported to be from $15 \%$ to $30 \%^{3}$ and its mechanism is multifactorial.

The gastrointestinal surgeon is confronted with the important aspect of decompressing the gastrointestinal tract following bowel surgery. It is especially significant in upper GI surgery. Bilateral truncal vagotomy performed during esophagectomy can lead to delayed gastric empting. When clinically significant delayed emptying occurs, it is associated with increased morbidity ${ }^{2}$ theoretically, a gastric drainage procedure should solve the problem. That is why pyloroplasty has been advocated as an adjunct to esophagectomy to avoid delayed gastric emptying. 4,5 However, it renders the pylorus incompetent and risks life-long bile reflux after esophagogastrectomy. Although a pyloromyotomy and pyloroplasty help the denervated stomach empty, there is often edema after this procedure, and this often prolongs gastric emptying in the early postoperative period when the patient is at the highest risk of aspiration. ${ }^{6}$
However, recent studies have challenged this decision, and it was shown that the function of the stomach is minimally impaired the formation of gastric tube used for reconstruction. ${ }^{1,7}$ Drainage procedure such as pyloroplasty or pyloromyotomy not consistently prevent delays gastric empting. They, however, may increase postoperative complications such additional sutureline leaks, orperforation when pyloromyotomy selected. In addition, it can lead tolong-termbilereflux, for which no satisfactory treatment. ${ }^{6}$ In another side, Zieren et al., ${ }^{8}$ showed that patients who undergo pyloroplasty can have a late pyloric stricture as well. Thus these procedures, although relatively safe, can lead to significant complications. ${ }^{8}$

The aim of our study is to demonstrate our experience in coping with this problem. It has been shown that Botulinum toxin (BT) type A is a potent inhibitor of acetylcholine release from presynaptic nerve endings ${ }^{6,9-12}$ and has been used in the treatment of spastic disorders, including smooth muscle disorders such as in Achalasia, gastroparesis. ${ }^{6,12-15}$ Botulinum toxin A selectively cleaves synaptosomal-associated protein 25 , which results in a failure of synaptic vesicles containing neurotransmitters undergo exocytosis and release of neurotransmitters. ${ }^{6,10}$ Synaptosomal associated protein 25 is also present in the gastrointestinal smooth muscle, suggesting additional site for botulinum toxin. However, the exact mechanism of botulinum toxin in the smooth muscles is currently unknown. ${ }^{6}$ We chose the same method, a less invasive and safer solution to the problem of delayed gastric emptying due to excessive activity of the pyloric muscle. 


\section{Material and methods}

Since 2003, data of all patients undergoing esophagectomy by a single surgeon (H.K.) has been collected in a concurrent fashion. A retrospective analysis of data was performed. We reviewed there cords of all patients who had Transhiatal esophagectomy without any drainage procedure of the pylorus. Decision not to perform drainage of pylorus received during surgery. Onlypylorus with increased muscletone had drainage procedure. All clinical data was recorded in an Excel (Microsoft Corporation) spread sheet. Postoperatively, our patients are out of bed and begin respiratory physical therapy on the first post-op day. All patients receive enteral feeding on the day following surgery via a feeding jejunostomy tube inserted during the operation as part of the surgical procedure. We keep the nasogastric (NG) tube for 2 to 5days, for drainage, while the patient is kept NPO (Nothing-per-os). The NG tube is withdrawn provided the drainage volume does not exceed $500 \mathrm{ml}$ of gastric fluid per $24 \mathrm{hrs}$. On this day, the patients are started of soft diet ad-lib. We do not routinely perform contrast swallow studies. If patients are observed to have increased amount of gastric content draining via the NG tube, they are suspected of developing delayed gastric empting. In these patients we continue with an esophagogram, using a water-soluble contrast material TelebrixR Gastro (MeglumineLoxatalamade) $300 \mathrm{mg} / \mathrm{ml}$. This test is known simple, available and enough diagnostic. This study usually confirms or excludes gastric outlet obstruction due to increased tonicity of the pyloric sphincter. Patients with this diagnosis were treated conservatively fasting, NGT, enteral nutrition via feeding jejunostomy for 14days. All patients had not improved during this period and were referred to the gastroenterologists and underwent endoscopic injection of botulinium toxin.

\section{Technique of endoscopic injection of botulinium toxin}

The procedure can be performed in an outpatient setting. Upper gastrointestinal endoscopy was performed under conscious sedation using a combination of Medazolam and Pethidine Hydrocloride. Standard monitoring is generally used during the endoscopic procedure. Following a complete endoscopic examination of the remaining stomach and duodenum, BT is injected using a standard a 25 gauge sclera therapy needle ( $5 \mathrm{~mm}$ long) that is passed through the accessory channel of the endoscope. The pylorus is identified, realizing that following this kind of surgery the pylorus has an oblique position and may be difficult to clearly visualize. To date, the optimal dosing of BT injection has not been determined. Botulinium toxin injection into the pyloric sphincter is reported to improve gastric emptying and reduce dyspeptic symptoms in patients with gastroparesis. ${ }^{6,14,15}$ In Achalasia, 100units of Botox have been injected. ${ }^{13}$ Up to 200units of BT in $5 \mathrm{ml}$ of $0.9 \%$ normal saline solution have been used in cases of intrapyloric injection during esophagectomy as a preventive procedure of delayed gastric empting. ${ }^{9}$ We chose to use Botulinium toxin (Allergan Inc., Irvin, California, USA) aliquots of 25units to be injected in four quadrants into the pyloric sphincter to a total of 100units. Following a brief post-procedure monitoring period and recovery, the patients are transferred to the floor. To date we have had no complications related to the injection of Botox.

\section{Results}

During the study period from 2003 to 2011, seventy-nine (79) patients underwent Transhiatal esophagectomy without pyloric manipulation. There were 57 males and 22 females with a mean age of 64 (range, 18-86). The operative mortality was 2.5 per cent (two patients: one from CVA, another from Hemorrhagic Pancreatitis). Indications for surgery included: Cancer-71 patients, Achalasia-4 patients and Caustic or Iatrogenic injury-4 patients (Table 1), of the 71 cancer patients, 33 received neo-adjuvant therapy due to locally advanced disease (T3N0-1). Cervical anastomosis was performed using a circular stapler(CDH, 25mm; Ethicon Endo-surgery, Johnson \& Johnson, Cincinnati, $\mathrm{OH}$ ) in 61 patients and manually (one layer, interrupted, PDS 4/0) in 18patients (Table 2) during the postoperative period twelve patients (15.4\%) developed cervical anastomosis leak, three patients after manual anastomosis and nine patients with circular stapler anastomosis. There was no mortality associated with leaks (Table 3). Three patients had an incidentals plenectomy due to iatrogenic injury during the surgical procedure. Five patients had a temporary unilateral injury to the Recurrent Laryngeal Nerve, which resolved with subsequent speech therapy. Five patients $(6.3 \%)$ developed postoperative gastric outlet obstruction due to pylorospasm. It is remarkable that none of the patients with pylorospasm developed anastomotic or staple line leaks. All were treated successfully with a single treatment of endoscopic Botox injections to the pyloric sphincter. There were no post-procedure signs of dysphagia or residual gastric emptying problems over a long term follow-up period (mean 9.6, range 1-25months) (Table 3).

Table I Demographic data

\begin{tabular}{ll}
\hline Number & 79 \\
Age & 64 (range 18-86). \\
Sex & 57 Males and 22 Females \\
Indication & \\
Cancer & 71 \\
Achalasia & 4 \\
Caustic and latrogenic & 4 \\
\hline
\end{tabular}

Table 2 Type of anastomosis

\begin{tabular}{ll}
\hline Anastomosis & \\
Stapler & 61 \\
Manual & 18
\end{tabular}

Table 3 Outcome data of patient

\begin{tabular}{ll}
\hline Anastomotic leak & $12(15.4 \%)$ \\
GOO* & $5(11.1 \%)$ \\
Mortality & $2(2.5 \%)$ \\
Botox injection & $5(6.3 \%)$ \\
Median follow up & 9.6, (range I-25months) \\
\hline
\end{tabular}

*Gastric outlet obstruction

\section{Discussion}

There is still no consensus among upper GI surgeons about whether to perform pyloroplasty or pyloromyotomy as an integral part of the transhiatal-esophagectomy. The concern is for delayed gastric emptying following this procedure which can lead to gastric dilatation and as a result, anastomotic breakdown with leakage and/ or aspiration. Aspiration and aspiration pneumonia are the most common and devastating early postoperative complications after esophagogastrectomy. ${ }^{6,9}, 16$ Many who prefer pyloroplasty argue 
that it helps gastric emptying associated with pyloric denervated and hence reduces the risk of pulmonary aspiration..$^{1,6,7}$ However, it renders the pylorus incompetent and risks life-long bile reflux after esophagogastrectomy. It can lead to long-term bile reflux, for which there is no satisfactory treatment. ${ }^{6}$ There are surgeons still advocate some kind of drainage procedures at the pylorus to prevent such dire complications. The literature suggests that gastric emptying procedures are to be beneficial, at least in terms of an earlier return to a normal diet and a possible reduction in respiratory complications. ${ }^{9}$ However, studies have shown that delayed gastric emptying can still persist despite a drainage procedure. Not to mention, that this common prophylactic procedure can lead to undesirable complications. Leak, stricture, bleeding, and death have been reported as complication of pyloroplasty. Physiological complication of an empting procedure may include bile reflux, dumping syndrome. Recent publications found that routine performance of pyloromyotomy or pyloroplasty may be associated with increased incidence of these symptoms without demonstration reduction in complications related to delay gastric empting., ${ }^{9}, 17$ There have been few prospective studies that have attempted to answer the question about the ideal pyloric evacuation procedure during resection of the esophagus and proximal stomach, but they were all either too little or disadvantage. ${ }^{6}$ Even been described by non-randomized retrospective study on 221patients, they have shown that the intraoperative injection of botulinum toxin in the pylorus during esophagogastrectomy is safe and effective and reduces the operation time of Ivor-Lewis operation. ${ }^{6}$ Fok et al., ${ }^{4}$ published the largest prospective randomized study on this issue. They randomized 200patients: 100 were pyloroplasty and 100 received no drainage procedure. All patients had used all of their stomachs, and they all underwent Lewis-Tanner operation. They reported, that 13 patients who did not receive drainage procedures were with gastric obstruction, 1 required reoperation, and 4 experienced pulmonary complications, of which 2 died. This study suggests that the pyloric drainage, in particular, pyloroplasty, it's better than no drainage for patients undergoing resection of the esophagus and proximal stomach, when the entire stomach channel is selected.,18 Mannell et al. ${ }^{19}$ showed in a prospective randomized study that pyloroplasty led to the dumping syndrome and the bile reflux. In additional, leaks pyloromyotomy or pyloroplasty may occur, and this adds another possible complication. Because it is often the gatekeeper at the diaphragm after esophagogastrectomy leak pyloromyotomy or pyloroplasty may be located in the chest, abdomen, or both, and so it may be difficult to manage. ${ }^{6}$ Considering recent reports we stopped any manipulation with pylorus during transhiatal esophagectomy. Our decision was supported by following reports. Palmes et al., ${ }^{17}$ reported on 198patients and concluded that "pyloric drainage after esophagectomy with gastric conduit reconstruction should be omitted because it does not improve gastric emptying and may favor biliary reflux esophagitis."

In our study, we clearly show that surgical drainage procedures can be safely excluded from the standard transhiatal esophagectomy, gastric pull-up and cervical anastomosis. Pylorospasm develops in a relatively small number of patients $(6.3 \%)$ and are not related with anastomotic leaks. Since, pyloromyotomy can add to the risk of complications, mainly and the fact that these procedures do not always prevent delayed gastric emptying, we suggest that there is no need for mandatory routine gastric drainage procedures in these patients. Post-operative endoscopic therapy with Botox injection to the pylorus can successfully resolve the problem of delayed gastric emptying in the few cases where pylorospasm develops.

\section{Acknowledgements}

None.

\section{Conflict of interest}

The author declares no conflict of interest.

\section{References}

1. Nguyen NT, Slone J, Wooldridge J, et al. Minimally invasive esophagectomy without the use of postoperative nasogastric tube decompression. Am Surg. 2009;75(10):929-931.

2. Aly A, Jamieson GG. Reflux after oesophagectomy. $\mathrm{Br} J$ Surg. 2004;91(2):137-141.

3. Lee HS, Kim MS, Lee JM, et al. Intrathoracic gastric emptying of solid food after esophagectomy for esophageal cancer. Ann ThoracSurg. 2005;80(2):443-447.

4. Fok M, Cheng SW, Wong J. Pyloroplasty versus no drainage in gastric replacement of the esophagus. Am J Surg. 1991;162(5):447-452.

5. Kao $\mathrm{CH}$, Chen $\mathrm{CY}$, Chen CL, et al. Gastric emptying of the intrathoracic stomach as oesophageal replacement for oesophageal carcinomas. $\mathrm{Nucl}$ Med Commun. 1994;15(3):152-155.

6. Cerfolio RJ1, Bryant AS, Canon CL, et al. Is botulinum toxin injection of the pylorus during Ivor-Lewis esophagogastrectomy the optimal drainage strategy? J Thorac Cardiovasc Surg. 2009;137(3):565-572.

7. Nguyen NT, Dholakia C, Nguyen XM, et al. Outcomes of minimally invasive esophagectomy without pyloroplasty:analysis of 109 cases. $\mathrm{Am}$ Surg. 2010;76(10):1135-1138.

8. Zieren HU, Muller JM, Jacobi CA, et al. Should a pyloroplasty be carried out in stomach transposition after subtotal esophagectomy with esophago-gastricanastomosis at the neck? A prospective randomized study. Chirurg. 1995;66(4):319-325.

9. Martin JT, Federico JA, Mckelvey AA, et al. Prevention of delayed gastric emptying after esophagectomy: a single center's experience with botulinum Toxin. Ann Thorac Surg. 2009;87(6):1708-1713.

10. Blasi J, Chapman ER, Link E, et al. Botulinum toxin A selectively cleaves the synaptic protein SNAP-25. Nature. 1993;365(6442):160163.

11. MacKenzie I, Burnstock G, Dolly JO. The effects of purified botulinum Neurotoxin type A on cholinergic, adrenergic and non-adrenergic, atropine resistant autonomic neuromuscular transmission. Neuroscience. 1982;7(4):997-1006.

12. Pasricha PJ, Ravich WJ, Hendrix TR, et al. Intrasphinctericbotulinum toxin for the treatment of Achalasia. N Engl J Med. 1995;332(12):774 778

13. Vaezi MF, Richter JE, Wilcox CM, et al. Botulinium toxin versus pneumatic dilatation in the treatment of Achalasia: a randomized trial. Gut. 1999;44(2):231-239.

14. Ezzedine D, Jit R, Katz N, et al. Pyloric injection of botulinum toxin for treatment of diabetic gastroparesis. Gastrointest Endosc. 2002;55(7):920-923.

15. Miller LS, Szych GA, Kantor SB, et al. Treatment of idiopathic gastroparesis with injection of botulinum toxin into the pyloric sphincter muscle. Am J Gastroenterol. 2002;97(7):1653-1660.

16. Kent MS, Pennathur A, Fabian T, et al. A pilot study of botulinum toxin injection for the treatment of delayed gastric emptying following esophagectomy. Surg Endosc. 2007;21(5):754-757. 
17. Palmes D, Weilinghoff M, Colombo-Benkmann M, et al. Effect of pyloric drainage procedures on gastric passage and bile reflux after esophagectomy with gastric conduit reconstruction. Langenbecks Arch Surg. 2007;392(2):135-141.

18. Ong GB, Kwong KH. The Lewis-Tanner operation for cancer of the oesophagus. J R Coll Surg Edinb. 1969;14(1):3-19.
19. Mannell A, Mcknight A, Esser JD. Role of pyloroplasty in the retrosternal stomach: results of a prospective, randomized, controlled trial. $\mathrm{Br} \mathrm{J}$ Surg. 1990;77(1):57-59. 